\title{
Some results on integral inequalities via Riemann-Liouville fractional integrals
}

\author{
Xiaoling Li' ${ }^{1}$, Shahid Qaisar ${ }^{2}$, Jamshed Nasir ${ }^{3}$, Saad Ihsan Butt ${ }^{3}$, Farooq Ahmad ${ }^{4,5^{*}}$ (D, Mehwish Bari ${ }^{6}$ and \\ Shan E Farooq ${ }^{7}$
}

\begin{tabular}{l}
\hline${ }^{\text {CCorrespondence: }}$ \\
ahmad.farooq@ntu.edu.sg; \\
farooqgujar@gmail.com \\
${ }^{4}$ School of Mechanical and \\
Aerospace Engineering, NANYANG \\
Technological University, Singapore, \\
Singapore \\
5 Punjab Higher Education \\
Department, Lahore, Pakistan \\
Full list of author information is \\
available at the end of the article
\end{tabular}

\section{Correspondence:}

farooggujar@gmail.com

${ }^{4}$ School of Mechanical and

Singapore

Full list of author information is

\begin{abstract}
In current continuation, we have incorporated the notion of $s-(\alpha, m)$-convex functions and have established new integral inequalities. In order to generalize Hermite-Hadamard-type inequalities, some new integral inequalities of Hermite-Hadamard and Simpson type using $s-(\alpha, m)$-convex function via Riemann-Liouville fractional integrals are obtained that reproduce the results presented by (Appl. Math. Lett. 11(5):91-95, 1998; Comput. Math. Appl. 47(2-3): 207-216, 2004; J. Inequal. Appl. 2013:158, 2013). Applications to special means are also provided.
\end{abstract}

MSC: $26 \mathrm{~A} 15 ; 26 \mathrm{~A} 51 ; 26 \mathrm{D} 10$

Keywords: Simpson's inequality; Convex functions; Power-mean inequality; Riemann-Liouville fractional integral

\section{Introduction}

Let $\mathbb{R}$ be the set of real numbers, $I \subseteq \mathbb{R}$ be an interval, and $\eta: I \subset \mathbb{R} \rightarrow \mathbb{R}$ be a convex in the classical sense function which satisfies the inequality

$$
\eta\left(\kappa s_{1}+(1-\kappa) s_{2}\right) \leq \kappa \eta\left(s_{1}\right)+(1-\kappa) \eta\left(s_{2}\right)
$$

whenever $s_{1}, s_{2} \in I$ and $\kappa \in[0,1]$. Numerous authors have presented inequalities for convex functions, however, because of its wide applicability and importance, one of the most notable is Hermite-Hadamard inequality, which is expressed as follows [4]:

Let $\eta: I \subset \mathbb{R} \rightarrow \mathbb{R}$ be a convex function on the interval $I$ of real numbers and $s_{1}, s_{2} \in I$ with $s_{1}<s_{2}$. Then

$$
\eta\left(\frac{s_{1}+s_{2}}{2}\right) \leq \frac{1}{s_{2}-s_{1}} \int_{s_{1}}^{s_{2}} g(t) d t \leq \frac{\eta\left(s_{1}\right)+\eta\left(s_{2}\right)}{2} .
$$

Both inequalities hold reversed if $\eta$ is concave. In the field of mathematical inequalities, Hermite-Hadamard inequalities have been considered by numerous mathematicians because of their pertinence and handiness. Various researchers have extended the HermiteHadamard inequality to different structures utilizing the classical convex functions. First we recall some important definitions and results which we have used in this paper.

M. Muddassar [5] presented the class of $s-(\alpha, m)$-convex functions as follows:

(c) The Author(s) 2019. This article is distributed under the terms of the Creative Commons Attribution 4.0 International License (http://creativecommons.org/licenses/by/4.0/), which permits unrestricted use, distribution, and reproduction in any medium, provided you give appropriate credit to the original author(s) and the source, provide a link to the Creative Commons license, and indicate if changes were made. 
Definition 1 A function $\eta:[0, \infty) \rightarrow[0, \infty)$ is said to be $s-(\alpha, m)$-convex in the first sense, or $f$ belongs to the class $K_{m, 1}^{\alpha, s}$, if for every $s_{1}, s_{2} \in[0, \infty]$ and $\kappa \in[0,1]$, the following inequality holds:

$$
\eta\left(\kappa s_{1}+m(1-\kappa) s_{2}\right) \leq \kappa^{\alpha s} \eta\left(s_{1}\right)+m\left(1-\kappa^{\alpha s}\right) \eta\left(s_{2}\right),
$$

where $(\alpha, m) \in[0,1]^{2}$, for some fixed $s \in(0,1]$.

Definition 2 A function $\eta:[0, \infty) \rightarrow[0, \infty)$ is said to be $s-(\alpha, m)$-convex function in the second sense, or $f$ belongs to the class $K_{m, 2}^{\alpha, s}$, if for every $s_{1}, s_{2} \in[0, \infty]$ and $\kappa \in[0,1]$, the following inequality holds:

$$
\eta\left(\kappa s_{1}+m(1-\kappa) s_{2}\right) \leq\left(\kappa^{\alpha}\right)^{s} \eta\left(s_{1}\right)+m\left(1-\kappa^{\alpha}\right)^{s} \eta\left(s_{2}\right),
$$

where $(\alpha, m) \in[0,1]^{2}$, for some fixed $s \in(0,1]$.

Definition 3 Let $f \in L_{1}[a, b]$. The left-sided and right-sided Riemann-Liouville fractional integrals of order $\alpha>0$, with $a \geq 0$, are defined by

$$
J_{a^{+}}^{\alpha} f(x)=\frac{1}{\Gamma(\alpha)} \int_{a}^{x}(x-t)^{\alpha-1} f(t) d t, \quad a<x,
$$

and

$$
J_{b-}^{\alpha} f(x)=\frac{1}{\Gamma(\alpha)} \int_{x}^{b}(t-x)^{\alpha-1} f(t) d t, \quad x<b
$$

respectively, where $\Gamma(\cdot)$ is the Gamma function defined by $\Gamma(\alpha)=\int_{0}^{\infty} e^{-u} u^{\alpha-1} d u$.

It is to be noted that $J_{a^{+}}^{0} f(x)=J_{b}^{0} f(x)=f(x)$. In the case of $\alpha=1$, the fractional integral reduces to the classical integral. Properties relating to this operator can be found in [6], and for useful details on Hermite-Hadamard and Simpson's type inequalities connected with fractional integral inequalities, the interested readers are directed to $[7,8]$.

In [1], Dragomir and Agarwal obtained inequalities for differentiable convex mappings which are connected with the right-hand side of Hermite-Hadamard (trapezoid) inequality and applied them to obtain some elementary inequalities for real numbers and in numerical integration.

Theorem 1 Let $\eta: I \subset R \rightarrow R$ be a differentiable mapping on $I$ where $s_{1}, s_{2} \in I$ with $s_{1}<s_{2}$. If $\left|\eta^{\prime}\right|^{q}$ is convex on $\left[s_{1}, s_{2}\right]$, for some $q \geq 1$, then the following inequality holds:

$$
\left|\frac{\eta\left(s_{1}\right)+\eta\left(s_{2}\right)}{2}-\frac{1}{s_{2}-s_{1}} \int_{s_{1}}^{s_{2}} \eta(u) d u\right| \leq \frac{s_{2}-s_{1}}{8}\left[\left|\eta^{\prime}\left(s_{1}\right)\right|+\left|\eta^{\prime}\left(s_{2}\right)\right|\right] .
$$

In [9], a variant of Hermite-Hadamard-type inequalities was obtained as follows.

Theorem 2 Let $\eta: I \subseteq \mathbb{R} \rightarrow \mathbb{R}$ be a differentiable function on I and let $s_{1}, s_{2} \in I$ with $s_{1}<s_{2}$. If $\left|\eta^{\prime}\right|$ is a convex function on $\left[s_{1}, s_{2}\right]$, then the following inequality holds:

$$
\left|\frac{1}{s_{2}-s_{1}} \int_{s_{1}}^{s_{2}} \eta(u) d u-\eta\left(\frac{s_{1}+s_{2}}{2}\right)\right| \leq \frac{s_{2}-s_{1}}{8}\left[\left|\eta^{\prime}\left(s_{1}\right)\right|+\left|\eta^{\prime}\left(s_{2}\right)\right|\right] .
$$


In [2], Yang obtained Hermite-Hadamard (trapezoid) inequalities for differentiable mapping for concave functions.

Theorem 3 Let $I \subset R$ bean open interval, $l, m, n, P, Q \in I$ with $l \leq P \leq n \leq Q \leq m(n \neq l, m)$ $l, m, n \in R$ and $\eta:\left[s_{1}, s_{2}\right] \rightarrow R$ be a differentiable function. If $\left|\eta^{\prime}\right|^{q}$ is concave on $\left[s_{1}, s_{2}\right]$, and $1 \leq \theta \leq q$, then

$$
\left|(P-l) \eta(l)+(m-Q) \eta(m)+(Q-P) \eta(n)-\int_{s_{1}}^{s_{2}} \eta(u) d u\right| \leq K(P, Q, n, \theta) J(P, Q, n, \theta)
$$

where

$$
K(P, Q, n, \theta)=\left(\frac{1}{2}\left[(P-l)^{2}+(n-P)^{2}+(Q-n)^{2}+(m-Q)^{2}\right]\right)^{\frac{(\theta-1)}{\theta}},
$$

and

$$
\begin{aligned}
J(P, Q, n, \theta) \\
=\left(\frac{1}{2}\left[(P-l)^{2}+(n-P)^{2}\right]\left|\eta^{\prime}\left(\frac{(P-l)^{2}+(n-P)^{2}(2 n-3 l+P)}{3\left[(P-l)^{2}+(n-P)^{2}\right]}+l\right)\right|^{\theta}\right)^{\frac{(\theta-1)}{\theta}} \\
\quad+\left(\frac{1}{2}\left[(Q-n)^{2}+(m-Q)^{2}\right]\left|\eta^{\prime}\left(m-\frac{(Q-n)^{2}(3 m-2 n-Q)+(m-Q)^{3}}{3\left[(Q-n)^{2}+(m-Q)^{2}\right]}\right)\right|^{\theta}\right)^{\frac{(\theta-1)}{\theta}} .
\end{aligned}
$$

Proposition 1 Under the assumptions of Theorem 3 with $P=Q=n=(l+m) / 2$ and $\theta=1$, we get the following inequality:

$$
\left|\frac{\eta\left(s_{1}\right)+\eta\left(s_{2}\right)}{2}-\frac{1}{s_{2}-s_{1}} \int_{s_{1}}^{s_{2}} \eta(u) d u\right| \leq \frac{s_{2}-s_{1}}{8}\left[\left|\eta^{\prime}\left(\frac{5 s_{1}+s_{2}}{6}\right)\right|+\left|\eta^{\prime}\left(\frac{s_{1}+5 s_{2}}{6}\right)\right|\right] .
$$

Proposition 2 Under the assumptions of Theorem 3 with $P=s_{1}, Q=s_{2}, n=(l+m) / 2$ and $\theta=1$, we get the following inequality:

$$
\left|\eta\left(\frac{s_{1}+s_{2}}{2}\right)-\frac{1}{s_{2}-s_{1}} \int_{s_{1}}^{s_{2}} \eta(u) d u\right| \leq \frac{s_{2}-s_{1}}{8}\left[\left|\eta^{\prime}\left(\frac{2 s_{1}+s_{2}}{3}\right)\right|+\left|\eta^{\prime}\left(\frac{s_{1}+2 s_{2}}{3}\right)\right|\right] .
$$

The aim of this paper is to build up Hermite-Hadamard-type inequalities for RiemannLiouville fractional integral using the $s-(\alpha, m)$ convexity, as well as concavity, for functions whose absolute values of the first derivative are convex. The results presented in this paper provide extension of those given in earlier works. The interested readers are referred to $[3,10-25]$.

\section{Main results}

In order to prove our main results, we need the following integral inequality

Lemma 1 Let $f:[a, b] \rightarrow \mathbb{R}$ be a differentiable function on $(a, b)$ with $a<b$, such that $f^{\prime}$ is integrable. Then the following inequality for Riemann-Liouville fractional integrals holds with $0<\alpha \leq 1$ :

$$
\left(1-\frac{2}{2^{\alpha}} \lambda\right) f\left(\frac{a+b}{2}\right)+\lambda \frac{f(a)+(b)}{2^{\alpha}}-\frac{\Gamma(\alpha+1)}{2(b-a)^{\alpha}}\left[J_{a^{+}}^{\alpha} f(b)+J_{b^{-}}^{\alpha} f(a)\right]
$$




$$
\leq \frac{b-a}{2^{\alpha+1}}\left[L_{1}+L_{2}+\left(L_{3}+L_{4}\right)\right]
$$

where $L_{1}=\int_{0}^{1}\left[(1-t)^{\alpha}-\lambda\right] f^{\prime}\left(t a+(1-t) \frac{a+b}{2}\right) d t, L_{2}=\int_{0}^{1}\left[\lambda-(1-t)^{\alpha}\right] f^{\prime}\left(t b+(1-t) \frac{a+b}{2}\right) d t$, $L_{3}=\int_{0}^{1}\left[2^{\alpha}-\lambda-(2-t)^{\alpha}\right] f^{\prime}\left(t \frac{a+b}{2}+(1-t) a\right) d t, L_{4}=\int_{0}^{1}\left[\lambda-2^{\alpha}+(2-t)^{\alpha}\right] f^{\prime}\left(t \frac{a+b}{2}+(1-t) b\right) d t$.

Proof A simple proof of this inequality can be obtained by integrating by parts. The details are left to the interested readers.

Using Lemma 1 the following results can be obtained.

Theorem 4 Let $f:[a, b] \rightarrow \mathbb{R}$ be a differentiable function on $(a, b)$ with $a<b$ such that $f^{\prime}$ is integrable. If $\left|f^{\prime}\right|$ is $s-(\alpha, m)$ convex on $[a, b]$, then the following inequality for RiemannLiouville fractional integrals holds with $0<\alpha \leq 1$ :

$$
\begin{aligned}
\mid(1 & \left.-\frac{2}{2^{\alpha}} \lambda\right) f\left(\frac{a+b}{2}\right)+\lambda \frac{f(a)+f(b)}{2^{\alpha}}-\frac{\Gamma(\alpha+1)}{2(b-a)^{\alpha}}\left[J_{a^{+}}^{\alpha} f(b)+J_{b^{-}}^{\alpha} f(a)\right] \mid \\
\leq & {\left[\left\{M_{1}\left|f^{\prime}(a)\right|+2 m\left(M_{2}-M_{1}\right)\left|f^{\prime}\left(\frac{a+b}{2}\right)\right|+M_{1}\left|f^{\prime}(b)\right|\right\}\right.} \\
& \left.+\left\{M_{3}\left|f^{\prime}(a)\right|+m\left(M_{4}-M_{3}\right)\left|f^{\prime}\left(\frac{a+b}{2}\right)\right|+M_{3}\left|f^{\prime}(b)\right|\right\}\right] \\
M_{1}= & \int_{0}^{1}\left|(1-t)^{\alpha}-\lambda\right| t^{\alpha s} d t=\frac{(\alpha s) !}{(\alpha s+s+1) !}-\frac{\lambda}{(s+1)}, \\
M_{2}= & \int_{0}^{1}\left|(1-t)^{\alpha}-\lambda\right| d t \\
= & \frac{1-2(1-\zeta)^{\alpha+1}}{\alpha+1}+(1-2 \zeta) \lambda, \\
M_{3}= & \int_{0}^{1}\left|2^{\alpha}-(2-t)^{\alpha}-\lambda\right| t^{\alpha s} d t \\
= & \frac{2^{\alpha s}}{s+1}-2^{\alpha s}\left[\frac{1}{(s+1)}-\frac{\alpha s}{2(s+1)}\right]-\frac{\lambda}{(s+1)}, \\
M_{4}= & \int_{0}^{1}\left|2^{\alpha}-(2-t)^{\alpha}-\lambda\right| d t \\
= & \frac{1+2^{\alpha+1}-2(2-\xi)^{\alpha+1}}{\alpha+1}+\left(2^{\alpha}-\lambda\right)(1-2 \xi),
\end{aligned}
$$

where

$$
\zeta=1-\lambda^{\frac{1}{\alpha}} \text { and } \xi=2-\left(2^{\alpha}-\lambda\right)^{\frac{1}{\alpha}}
$$

Proof Using $s-(\alpha, m)$ convexity of $\left|f^{\prime}\right|$, for all $t \in[0,1]$, we obtain:

$$
\begin{aligned}
\left|L_{1}\right| & \leq \int_{0}^{1}\left((1-t)^{\alpha}-\lambda\right)\left|f^{\prime}\left(t a+(1-t) \frac{a+b}{2}\right)\right| d t \\
& =\int_{0}^{1}\left((1-t)^{\alpha}-\lambda\right)\left|t^{\alpha s} f^{\prime}(a)+m\left(1-t^{\alpha s}\right) f^{\prime}\left(\frac{a+b}{2}\right)\right| d t
\end{aligned}
$$




$$
\begin{aligned}
& \leq \int_{0}^{1}\left((1-t)^{\alpha}-\lambda\right)\left\{t^{\alpha s}\left|f^{\prime}(a)\right|+m\left(1-t^{\alpha s}\right)\left|f^{\prime}\left(\frac{a+b}{2}\right)\right|\right\} d t \\
& =M_{1}\left|f^{\prime}(a)\right|+m\left(M_{2}-M_{1}\right)\left|f^{\prime}\left(\frac{a+b}{2}\right)\right| \text {, } \\
& \left|L_{2}\right| \leq \int_{0}^{1}\left((1-t)^{\alpha}-\lambda\right)\left|f^{\prime}\left(t b+(1-t) \frac{a+b}{2}\right)\right| d t \\
& =\int_{0}^{1}\left((1-t)^{\alpha}-\lambda\right)\left|t^{\alpha s} f^{\prime}(b)+m\left(1-t^{\alpha s}\right) f^{\prime}\left(\frac{a+b}{2}\right)\right| d t \\
& \leq \int_{0}^{1}\left((1-t)^{\alpha}-\lambda\right)\left\{t^{\alpha s}\left|f^{\prime}(b)\right|+m\left(1-t^{\alpha s}\right)\left|f^{\prime}\left(\frac{a+b}{2}\right)\right|\right\} d t \\
& =M_{1}\left|f^{\prime}(b)\right|+m\left(M_{2}-M_{1}\right)\left|f^{\prime}\left(\frac{a+b}{2}\right)\right| \text {, } \\
& \left|L_{3}\right|=\int_{0}^{1}\left(2^{\alpha}-(2-t)^{\alpha}-\lambda\right)\left\{\left|f^{\prime}\left(t \frac{a+b}{2}+(1-t) a\right)\right|\right\} d t \\
& =\int_{0}^{1}\left(2^{\alpha}-(2-t)^{\alpha}-\lambda\right)\left\{\left|t^{\alpha s} f^{\prime}\left(\frac{a+b}{2}\right)+m\left(1-t^{\alpha s}\right) f^{\prime}(a)\right|\right\} d t \\
& \leq \int_{0}^{1}\left(2^{\alpha}-(2-t)^{\alpha}-\lambda\right)\left\{t^{\alpha s}\left|f^{\prime}\left(\frac{a+b}{2}\right)\right|+m\left(1-t^{\alpha s}\right)\left|f^{\prime}(a)\right|\right\} d t \\
& =M_{3}\left|f^{\prime}\left(\frac{a+b}{2}\right)\right|+m\left(M_{4}-M_{3}\right)\left|f^{\prime}(a)\right| \text {, } \\
& \left|L_{4}\right| \leq \int_{0}^{1}\left(2^{\alpha}-(2-t)^{\alpha}-\lambda\right)\left\{\left|f^{\prime}\left(t \frac{a+b}{2}+(1-t) b\right)\right|\right\} d t \\
& =\int_{0}^{1}\left(2^{\alpha}-(2-t)^{\alpha}-\lambda\right)\left\{\left|t^{\alpha s} f^{\prime}\left(\frac{a+b}{2}\right)+m\left(1-t^{\alpha s}\right) f^{\prime}(b)\right|\right\} d t \\
& \leq \int_{0}^{1}\left(2^{\alpha}-(2-t)^{\alpha}-\lambda\right)\left\{t^{\alpha s}\left|f^{\prime}\left(\frac{a+b}{2}\right)\right|+m\left(1-t^{\alpha s}\right)\left|f^{\prime}(b)\right|\right\} d t \\
& =M_{3}\left|f^{\prime}\left(\frac{a+b}{2}\right)\right|+m\left(M_{4}-M_{3}\right)\left|f^{\prime}(b)\right| \text {. }
\end{aligned}
$$

Corollary 1 Under the assumptions Theorem 4, with $\alpha=s=m=1$,

$$
\begin{aligned}
& \left|(1-\lambda) f\left(\frac{a+b}{2}\right)+\lambda \frac{f(a)+f(b)}{2}-\frac{1}{b-a} \int_{a}^{b} f(x) d x\right| \\
& \quad \leq \frac{b-a}{8}\left[2 \lambda^{2}-2 \lambda+1\right]\left(\left|f^{\prime}(a)\right|+\left|f^{\prime}(b)\right|\right) .
\end{aligned}
$$

Remark 1 Taking $\lambda=1$, inequality (7) reduces to inequality (1).

Remark 2 Taking $\lambda=0$, inequality (7) reduces to inequality (2).

The corresponding version for powers of the absolute value of the derivative is incorporated in the following theorem. 
Theorem 5 Let $f$ be defined as in Theorem 4 and suppose $\left|f^{\prime}\right|^{q}$ is a convex on $[a, b]$, with $q \geq 1$. Then the following inequality holds:

$$
\begin{aligned}
\mid(1 & \left.-\frac{2}{2^{\alpha}} \lambda\right) f\left(\frac{a+b}{2}\right)+\lambda \frac{f(a)+f(b)}{2^{\alpha}}-\frac{\Gamma(\alpha+1)}{2(b-a)^{\alpha}}\left[J_{a^{+}}^{\alpha} f(b)+J_{b-}^{\alpha} f(a)\right] \mid \\
\leq & \frac{(b-a)}{2^{\alpha}} \\
& \times\left[( M _ { 2 } ) ^ { 1 - 1 / q } \left\{\left(M_{1}\left|f^{\prime}(b)\right|^{q}+m\left(M_{2}-M_{1}\right)\left|f^{\prime}\left(\frac{a+b}{2}\right)\right|^{q}\right)^{1 / q}\right.\right. \\
& \left.+\left(M_{1}\left|f^{\prime}(a)\right|^{q}+m\left(M_{2}-M_{1}\right)\left|f^{\prime}\left(\frac{a+b}{2}\right)\right|^{q}\right)^{1 / q}\right\} \\
& +\left(M_{4}\right)^{1-1 / q}\left\{\left(M_{3}\left|f^{\prime}(b)\right|^{q}+m\left(M_{4}-M_{3}\right)\left|f^{\prime}\left(\frac{a+b}{2}\right)\right|^{q}\right)^{1 / q}\right. \\
& \left.\left.+\left(M_{3}\left|f^{\prime}(b)\right|^{q}+m\left(M_{4}-M_{3}\right)\left|f^{\prime}\left(\frac{a+b}{2}\right)\right|^{q}\right)^{1 / q}\right\}\right] .
\end{aligned}
$$

Proof Using the well-known power-mean integral inequality for $q>1$ and convexity of $\left|f^{\prime}\right|^{q}$, we have

$$
\begin{aligned}
\left|L_{1}\right| \leq & \left(\int_{0}^{1}\left|\left((1-t)^{\alpha}-\lambda\right)\right| d t\right)^{1-1 / q}\left(\left.\int_{0}^{1}\left|\left((1-t)^{\alpha}-\lambda\right)\right| f^{\prime}\left(t a+(1-t) \frac{a+b}{2}\right)\right|^{q} d t\right)^{1 / q} \\
\leq & \left(\int_{0}^{1}\left|\left((1-t)^{\alpha}-\lambda\right)\right| d t\right)^{1-1 / q} \\
& \times\left(\int_{0}^{1}\left|\left((1-t)^{\alpha}-\lambda\right)\right|\left\{t^{\alpha s}\left|f^{\prime}(a)\right|^{q}+m\left(1-t^{\alpha s}\right)\left|f^{\prime}\left(\frac{a+b}{2}\right)\right|^{q}\right\} d t\right)^{1 / q} \\
\leq & \left(M_{2}\right)^{1-1 / q}\left(M_{1}\left|f^{\prime}(a)\right|^{q}+m\left(M_{2}-M_{1}\right)\left|f^{\prime}\left(\frac{a+b}{2}\right)\right|^{q}\right)^{1 / q}, \\
\left|L_{2}\right| \leq & \left.\left(\int_{0}^{1}\left|\left((1-t)^{\alpha}-\lambda\right)\right| d t\right)\right)^{1-1 / q} \\
& \times\left(\int_{0}^{1}\left|\left((1-t)^{\alpha}-\lambda\right)\right| d t\left|f^{\prime}\left(t b+(1-t) \frac{a+b}{2}\right)\right|^{q} d t\right)^{1 / q} \\
\leq & \left.\left(\int_{0}^{1}\left|\left((1-t)^{\alpha}-\lambda\right)\right| d t\right)\right)^{1-1 / q} \\
& \times\left(\int_{0}^{1}\left|\left((1-t)^{\alpha}-\lambda\right)\right| d t\left\{t^{\alpha s}\left|f^{\prime}(b)\right|^{q}+m\left(1-t^{\alpha s}\right)\left|f^{\prime}\left(\frac{a+b}{2}\right)\right|^{q}\right\} d t\right)^{1 / q} \\
\leq & \left(M_{2}\right)^{1-1 / q}\left(M_{1}\left|f^{\prime}(b)\right|^{q}+m\left(M_{2}-M_{1}\right)\left|f^{\prime}\left(\frac{a+b}{2}\right)\right|^{q}\right)^{1 / q}, \\
\leq & \left(\int_{0}^{1}\left|2^{\alpha}-(2-t)^{\alpha}-\lambda\right| d t\right)^{1-1 / q} \\
\left|L_{3}\right| \leq & \left(\int_{0}^{1}\left|2^{\alpha}-(2-t)^{\alpha}-\lambda\right| d t\right)^{1-1 / q} \\
& \times\left(\left.\int_{0}^{1}\left|2^{\alpha}-(2-t)^{\alpha}-\lambda\right| f^{\prime}\left(t \frac{a+b}{2}+(1-t) a\right)\right|^{q} d t\right)^{1 / q} \\
&
\end{aligned}
$$




$$
\begin{aligned}
& \times\left(\int_{0}^{1}\left|2^{\alpha}-(2-t)^{\alpha}-\lambda\right|\left\{t^{\alpha s}\left|f^{\prime}\left(\frac{a+b}{2}\right)\right|^{q}+m\left(1-t^{\alpha s}\right)\left|f^{\prime}(a)\right|^{q}\right\} d t\right)^{1 / q} \\
\leq & \left(M_{4}\right)^{1-1 / q}\left(M_{3}\left|f^{\prime}\left(\frac{a+b}{2}\right)\right|^{q}+m\left(M_{4}-M_{3}\right)\left|f^{\prime}(a)\right|^{q}\right)^{1 / q}, \\
\left|L_{4}\right| \leq & \left(\int_{0}^{1}\left|2^{\alpha}-(2-t)^{\alpha}-\lambda\right| d t\right)^{1-1 / q} \\
& \times\left(\int_{0}^{1}\left|2^{\alpha}-(2-t)^{\alpha}-\lambda\right|\left|f^{\prime}\left(t \frac{a+b}{2}+(1-t) b\right)\right|^{q} d t\right)^{1 / q} \\
\leq & \left(\int_{0}^{1}\left|2^{\alpha}-(2-t)^{\alpha}-\lambda\right| d t\right)^{1-1 / q} \\
& \times\left(\int_{0}^{1}\left|2^{\alpha}-(2-t)^{\alpha}-\lambda\right|\left\{t^{\alpha s}\left|f^{\prime}\left(\frac{a+b}{2}\right)\right|^{q}+m\left(1-t^{\alpha s}\right)\left|f^{\prime}(b)\right|^{q}\right\} d t\right)^{1 / q} \\
\leq & \left(M_{4}\right)^{1-1 / q}\left(M_{3}\left|f^{\prime}\left(\frac{a+b}{2}\right)\right|^{q}+m\left(M_{4}-M_{3}\right)\left|f^{\prime}(b)\right|^{q}\right)^{1 / q} \cdot
\end{aligned}
$$

This completes the proof.

Corollary 2 Under the assumptions Theorem 5 , with $\alpha=s=m=1, \lambda=0$ in inequality (8), the following inequality holds:

$$
\begin{aligned}
& \left|f\left(\frac{a+b}{2}\right)-\frac{1}{b-a} \int_{a}^{b} f(x) d x\right| \\
& \quad \leq \frac{b-a}{8}\left[\left(\frac{1}{3}\left|f^{\prime}(a)\right|+\frac{2}{3}\left|f^{\prime}\left(\frac{a+b}{2}\right)\right|^{q}\right)^{\frac{1}{q}}+\left(\frac{1}{3}\left|f^{\prime}(b)\right|+\frac{2}{3}\left|f^{\prime}\left(\frac{a+b}{2}\right)\right|^{q}\right)^{\frac{1}{q}}\right] .
\end{aligned}
$$

Corollary 3 Under the assumptions Theorem 5 , with $\alpha=s=m=1, \lambda=1$ in inequality (8), the following inequality holds:

$$
\begin{aligned}
& \left|\frac{f(a)+f(b)}{2}-\frac{1}{b-a} \int_{a}^{b} f(x) d x\right| \\
& \quad \leq \frac{b-a}{8}\left[\left(\frac{2}{3}\left|f^{\prime}(a)\right|+\frac{1}{3}\left|f^{\prime}\left(\frac{a+b}{2}\right)\right|^{q}\right)^{\frac{1}{q}}+\left(\frac{2}{3}\left|f^{\prime}(b)\right|+\frac{1}{3}\left|f^{\prime}\left(\frac{a+b}{2}\right)\right|^{q}\right)^{\frac{1}{q}}\right] .
\end{aligned}
$$

Corollary 4 Under the assumptions Theorem 5 , with $\alpha=s=m=1, \lambda=\frac{1}{3}$ in inequality (8), the following inequality holds:

$$
\begin{gathered}
\left|\frac{1}{3}\left\{2 f\left(\frac{a+b}{2}\right)+\frac{f(a)+f(b)}{2}\right\}-\frac{1}{b-a} \int_{a}^{b} f(x) d x\right| \\
\leq \frac{5(b-a)}{72} \times\left[\left(\frac{16}{45}\left|f^{\prime}(a)\right|^{q}+\frac{29}{45}\left|f^{\prime}\left(\frac{a+b}{2}\right)\right|^{q}\right)^{\frac{1}{q}}\right. \\
\left.+\left(\frac{16}{45}\left|f^{\prime}(b)\right|^{q}+\frac{29}{45}\left|f^{\prime}\left(\frac{a+b}{2}\right)\right|^{q}\right)^{\frac{1}{q}}\right] .
\end{gathered}
$$

Theorem 6 Let $f:[a, b] \rightarrow \mathbb{R}$ be a differentiable function on $(a, b)$ with $a<b$ such that $f^{\prime}$ is integrable. If $\left|f^{\prime}\right|^{q}$ is concave on $[a, b]$, then the following inequality for Riemann-Liouville 
fractional integrals holds with $0<\alpha \leq 1$ :

$$
\begin{aligned}
(1- & \left.\frac{2}{2^{\alpha}} \lambda\right) f\left(\frac{a+b}{2}\right)+\lambda \frac{f(a)+f(b)}{2^{\alpha}}-\frac{\Gamma(\alpha+1)}{2(b-a)^{\alpha}}\left[J_{a^{+}}^{\alpha} f(b)+J_{b^{-}}^{\alpha} f(a)\right] \\
\leq & \frac{(b-a)}{2^{\alpha+1}} \times\left[M_{2}\left\{\left|f^{\prime}\left(\frac{M_{5} a+\left(M_{2}-M_{5}\right)\left(\frac{a+b}{2}\right)}{M_{2}}\right)\right|+\left|f^{\prime}\left(\frac{M_{5} b+\left(M_{2}-M_{5}\right)\left(\frac{a+b}{2}\right)}{M_{2}}\right)\right|\right\}\right. \\
& \left.+M_{4}\left|f^{\prime}\left(\frac{M_{6}\left(\frac{a+b}{2}\right)+\left(M_{4}-M_{6}\right) a}{M_{4}}\right)\right|+\left|f^{\prime}\left(\frac{M_{6}\left(\frac{a+b}{2}\right)+\left(M_{4}-M_{6}\right) b}{M_{4}}\right)\right|\right] \\
M_{5}= & \int_{0}^{1}\left|(1-t)^{\alpha}-\lambda\right| t d t \\
= & \frac{1-2(1-\zeta)^{\alpha+2}}{(\alpha+1)(\alpha+2)}-\frac{2 \zeta(1-\zeta)^{\alpha+1}}{\alpha+1}+\frac{\lambda}{2}\left(1-2 \zeta^{2}\right), \\
M_{6}= & \int_{0}^{1}\left|2^{\alpha}-(2-t)^{\alpha}-\lambda\right| t d t \\
= & \frac{1+2^{\alpha+2}-2(2-\xi)^{\alpha+2}}{(\alpha+1)(\alpha+2)}+\frac{1-2 \xi(2-\xi)^{\alpha+1}}{\alpha+1}+\left(2^{\alpha}-\lambda\right)\left(\frac{1}{2}-\xi^{2}\right) .
\end{aligned}
$$

Proof Using the concavity of $\left|f^{\prime}\right|^{q}$ and the power-mean inequality, we obtain

$$
\begin{aligned}
\left|f^{\prime}(\lambda a+(1-\lambda) b)\right|^{q} & >\lambda\left|f^{\prime}(a)\right|^{q}+(1-\lambda)\left|f^{\prime}(b)\right|^{q} \\
& \geq\left(\lambda\left|f^{\prime}(a)\right|+(1-\lambda) f^{\prime}(b) \mid\right)^{q} .
\end{aligned}
$$

Hence

$$
\left|f^{\prime}(\lambda a+(1-\lambda) b)\right| \geq \lambda\left|f^{\prime}(a)\right|+(1-\lambda)\left|f^{\prime}(b)\right|,
$$

so $\left|f^{\prime}\right|$ is also concave. By the Jensen integral inequality, we have

$$
\begin{aligned}
(1- & \left.\frac{2}{2^{\alpha}} \lambda\right) f\left(\frac{a+b}{2}\right)+\lambda \frac{f(a)+f(b)}{2^{\alpha}}-\frac{\Gamma(\alpha+1)}{2(b-a)^{\alpha}}\left[J_{a^{+}}^{\alpha} f(b)+J_{b^{-}}^{\alpha} f(a)\right] \\
\leq & \left(\int_{0}^{1}\left|\left((1-t)^{\alpha}-\lambda\right)\right| d t\right)\left|f^{\prime}\left(\frac{\int_{0}^{1}\left|\left((1-t)^{\alpha}-\lambda\right)\right|\left(t a+(1-t) \frac{a+b}{2}\right) d t}{\int_{0}^{1}\left|\left((1-t)^{\alpha}-\lambda\right)\right| d t}\right)\right| \\
& +\left(\int_{0}^{1}\left|\left((1-t)^{\alpha}-\lambda\right)\right| d t\right)\left|f^{\prime}\left(\frac{\int_{0}^{1}\left|\left((1-t)^{\alpha}-\lambda\right)\right|\left(t b+(1-t) \frac{a+b}{2}\right) d t}{\int_{0}^{1}\left|\left((1-t)^{\alpha}-\lambda\right)\right| d t}\right)\right| \\
& +\left(\int_{0}^{1}\left|\left(2^{\alpha}-(2-t)^{\alpha}-\lambda\right)\right| d t\right)\left|f^{\prime}\left(\frac{\int_{0}^{1}\left|\left(2^{\alpha}-(2-t)^{\alpha}-\lambda\right)\right|\left(t \frac{a+b}{2}+(1-t) a d t\right.}{\int_{0}^{1}\left|\left(2^{\alpha}-(2-t)^{\alpha}-\lambda\right)\right| d t}\right)\right| \\
& +\left(\int_{0}^{1}\left|\left(2^{\alpha}-(2-t)^{\alpha}-\lambda\right)\right| d t\right)\left|f^{\prime}\left(\frac{\int_{0}^{1}\left|\left(2^{\alpha}-(2-t)^{\alpha}-\lambda\right)\right|\left(t \frac{a+b}{2}+(1-t) b d t\right.}{\int_{0}^{1}\left|\left(2^{\alpha}-(2-t)^{\alpha}-\lambda\right)\right| d t}\right)\right|, \\
(1- & \left.\frac{2}{2^{\alpha}} \lambda\right) f\left(\frac{a+b}{2}\right)+\lambda \frac{f(a)+f(b)}{2^{\alpha}}-\frac{\Gamma(\alpha+1)}{2(b-a)^{\alpha}}\left[J_{a^{\alpha}}^{\alpha} f(b)+J_{b-}^{\alpha} f(a)\right] \\
= & M_{2}\left|f^{\prime}\left(\frac{M_{5} a+\left(M_{2}-M_{5}\right)\left(\frac{a+b}{2}\right)}{M_{2}}\right)\right|+M_{2}\left|f^{\prime}\left(\frac{M_{5} b+\left(M_{2}-M_{5}\right)\left(\frac{a+b}{2}\right)}{M_{2}}\right)\right|
\end{aligned}
$$




$$
+M_{4}\left|f^{\prime}\left(\frac{M_{6}\left(\frac{a+b}{2}\right)+\left(M_{4}-M_{6}\right) a}{M_{4}}\right)\right|+M_{4}\left|f^{\prime}\left(\frac{M_{6}\left(\frac{a+b}{2}\right)+\left(M_{4}-M_{6}\right) b}{M_{4}}\right)\right| .
$$

Remark 3 Under the assumptions Theorem 6, with $\alpha=s=1, \lambda=0$ in inequality (9), we obtain inequality (4).

Remark 4 Under the assumptions Theorem 6, with $\alpha=s=1, \lambda=1$ in inequality (9), we obtain inequality (5).

Corollary 5 Under the assumptions Theorem 6, with $\alpha=s=1, \lambda=\frac{1}{3}$ in inequality (9), the following inequality holds:

$$
\begin{aligned}
& \left|\left[\frac{1}{6} f(a)+\frac{2}{3} f\left(\frac{a+b}{2}\right)+\frac{1}{6} f(b)\right]-\frac{1}{b-a} \int_{a}^{b} f(x) d x\right| \\
& \quad \leq \frac{5(b-a)}{72}\left[\left|f^{\prime}\left(\frac{29 a+61 b}{90}\right)\right|+\left|f^{\prime}\left(\frac{61 a+29 b}{90}\right)\right|\right] .
\end{aligned}
$$

Remark 5 Inequality (10) is a generalization of the inequality obtained in [3, Theorem 8].

\section{Applications to special means}

We consider some means for arbitrary positive real numbers $a$ and $b$ (see, for instance, [4]):

- The arithmetic mean

$$
A=A(a, b)=\frac{a+b}{2}, \quad a, b \in \mathbb{R} \text { with } a, b>0 ;
$$

- The geometric mean

$$
G=G(a, b)=\sqrt{a b}, \quad a, b \in \mathbb{R} \text { with } a, b>0 ;
$$

- The harmonic mean

$$
H=H(a, b)=\frac{2 a b}{a+b}, \quad a, b \in \mathbb{R} \backslash\{0\} ;
$$

- The identric mean

$$
I=I(a, b)= \begin{cases}a & \text { if } a=b, \\ \frac{1}{e}\left(\frac{b^{b}}{a^{a}}\right) \frac{1}{b-a} & \text { if } a \neq b, a, b>0 ;\end{cases}
$$

- The logarithmic mean

$$
L=L(a, b)= \begin{cases}a & \text { if } a=b \\ \frac{b-a}{\ln b-\ln a} & \text { if } a \neq b\end{cases}
$$

- Generalized logarithmic mean

$$
L_{n}(a, b)= \begin{cases}a & \text { if } a=b, \\ {\left[\frac{b^{n+1}-a^{n+1}}{(n+1)(b-a)}\right]^{\frac{1}{n}}} & \text { if } a \neq b, n \in \mathbb{Z} \backslash\{-1,0\}, a, b>0 .\end{cases}
$$


Proposition 3 Let $n \in \mathbb{Z} \backslash\{-1,0\}$ and $a, b>0$. Then we have the following inequality:

$$
\left|(1-\lambda) A^{n}(a, b)+\lambda A\left(a^{n}, b^{n}\right)-L_{n}^{n}(a, b)\right| \leq \frac{n(b-a)}{4}\left(2 \lambda^{2}-2 \lambda+1\right) A\left(\left|a^{n-1}\right|,\left|b^{n-1}\right|\right) .
$$

For $\lambda=1$, we have

$$
\left|\lambda A\left(a^{n}, b^{n}\right)-L_{n}^{n}(a, b)\right| \leq \frac{n(b-a)}{4}\left(2 \lambda^{2}-2 \lambda+1\right) A\left(\left|a^{n-1}\right|,\left|b^{n-1}\right|\right) .
$$

For $\lambda=0$, we have

$$
\left|\lambda A^{n}\left(a^{n}, b^{n}\right)-L_{n}^{n}(a, b)\right| \leq \frac{n(b-a)}{4}\left(2 \lambda^{2}-2 \lambda+1\right) A\left(\left|a^{n-1}\right|,\left|b^{n-1}\right|\right) .
$$

Proof The assertion follows from Corollary 1 for $f(x)=x^{n}$ and with $n$ as specified above

Proposition 4 For all $0<a \leq b$, we have the following inequality:

$$
|\ln [A(1+a, 1+b)] I(1+b, 1+a)| \leq \frac{2(b-a)}{3^{1+\frac{1}{q}}}\left(\frac{1}{1+a}+\frac{2}{A^{q}(1+b, 1+a)}\right)^{\frac{1}{q}} .
$$

Proof The first assertion follows from Corollary 2 for $f(x)=-\ln (1+x) x^{n}$.

$$
|\ln [G(1+a, 1+b)] I(1+b, 1+a)| \leq \frac{2(b-a)}{3^{1+\frac{1}{q}}}\left(\frac{1}{1+a}+\frac{2}{A^{q}(1+b, 1+a)}\right)^{\frac{1}{q}} .
$$

The second assertion follows from Corollary 3 for $f(x)=-\ln (1+x)$.

Let $n \in \mathbb{Z} \backslash\{-1,0\} ; a, b>0$ then we have the following inequality

$$
\left|2 A^{n}(a, b)+A\left(a^{n}, b^{n}\right)-3 L(a, b)\right| \leq \frac{5 n(b-a)}{12} \frac{\left[29 A^{q(n-1)}(a, b)+16 b^{q(n-1)}\right]}{45^{\frac{1}{q}}} .
$$

The third assertion follows from Corollary 4 for $f(x)=x^{n}$ and as $n$ as specified above.

\section{Acknowledgements}

The second author is grateful to Prof. Dr. S.M. Junaid Zaidi, Executive Director and Prof. Dr. Raheel Qamar, Rector, COMSATS University Islamabad, Sahiwal Campus, Pakistan, for providing excellent research facilities. The fifth author is grateful to Punjab HEC, Lahore, Pakistan, also of Prof. Dr. Ooi and Prof. Dr. Leong, MAE, NTU, Singapore for providing excellent research facilities.

\section{Funding}

This research [Grant No.5325/Federal/NRPU/R\&D/HEC/2016] was partially supported by the Higher Education Commission of Pakistan.

\section{Competing interests}

Authors have declared no conflict of interest.

\section{Authors' contributions}

Dr. SQ and Dr. JN analyzed the problem and suggested mathematical modeling. Dr. SIB and Dr. MB generalized the results by proposing various lemmas. This manuscript has been written by SEF, Prof. Dr. FA and Prof. Dr. XL. Both the mentioned professors also made some necessary corrections regarding mathematical formulations and response to the reviewer. All authors had read and approved the final manuscript.

\section{Author details}

'School of Information Science and Engineering, Chengdu University, Chengdu, China. ${ }^{2}$ Department of Mathematics, COMSATS University Islamabad, Sahiwal Campus, Pakistan. ${ }^{3}$ Department of Mathematics, COMSATS University Islamabad, Lahore Campus, Pakistan. ${ }^{4}$ School of Mechanical and Aerospace Engineering, NANYANG Technological University, Singapore, Singapore. ${ }^{5}$ Punjab Higher Education Department, Lahore, Pakistan. ${ }^{6}$ Department of Mathematics, NCBA\&E, Bahawalpur, Pakistan. ${ }^{7}$ Department of Mathematics, GC University, Lahore, Pakistan. 


\section{Publisher's Note}

Springer Nature remains neutral with regard to jurisdictional claims in published maps and institutional affiliations.

Received: 1 February 2019 Accepted: 17 July 2019 Published online: 06 August 2019

\section{References}

1. Dragomir, S.-S., Agarwal, R.-P.: Two inequalities for differentiable mapping and applications to special means of real numbers and to trapezoidal formula. Appl. Math. Lett. 11(5), 91-95 (1998)

2. Yang, G.-S., Hwang, D.-Y., Tseng, K.-L.: Some inequalities for differentiable convex and concave mapping. Comput. Math. Appl. 47(2-3), 207-216 (2004)

3. Qaisar, S., He, C., Hussain, S.: A generalizations of Simpson's type inequality for differentiable functions using $(\alpha, m)$-convex functions and applications. J. Inequal. Appl. 2013, Article ID 158 (2013)

4. Dragomir, S.S., Pearce, C.-E.-M.: Selected topics on Hermite-Hadamard inequalities and applications, RGMIA Monographs, Victoria University (2000)

5. Muddassar, M., Bhatti, M.-I., Irshad, W.: Generalisation of integral inequalities of Hermite-Hadamard type through convexity. Bull. Aust. Math. Soc. 88(2), 320-330 (2014)

6. Gorenflo, R., Mainard, F.: Fractional calculus: integral and differential equations of fractional order. In: Fractals and Fractional Calculus in Continuum Mechanics, pp. 223-276. Springer, Wien (1997)

7. Belarbi, S., Dahmani, Z:: On some new fractional integral inequalities. J. Inequal. Pure Appl. Math. 10(3), 86 (2009)

8. Dahmani, Z: New inequalities in fractional integrals. Int. J. Nonlinear Sci. 9(4), 493-497 (2010)

9. KIrmaci, U.-S.: Inequalities for differentiable mappings and applications to special means of real numbers and to midpoint formula. Appl. Math. Comput. 147, 137-146 (2004)

10. Hwang, S.R., Tseng, K.-L., Hsu, K.-C.: New inequalities for fractional integrals and their applications. Turk. J. Math. 40, 471-486 (2016)

11. Iqbal, M., Qaisar, S., Muddassar, M.: A short note on integral inequality of Hermite-Hadamard type through convexity. J. Comput. Anal. Appl. 21(5), 946-953 (2016)

12. Iqbal, M., Qaisar, S., Hussain, S.: On Simpson's type inequalities utilizing fractional integrals. J. Comput. Anal. Appl. 23(6), 1137-1145 (2016)

13. Qaisar, S., labal, M., Muddassar, M.: New Hermite-Hadamard's inequalities for preinvex function via fractional integrals. J. Comput. Anal. Appl. 20(7), 1318-1328 (2016)

14. Sarikaya, M.-Z., Budak, H.: Generalized Hermite-Hadamard type integral inequalities for fractional integrals. Filomat 30(5), 1315-1326 (2016)

15. Qaisar, S., Gujjar, M.-F., Dragomir, S.-S., Iqbal, M.: New Hermite-Hadamard's inequalities via fractional integrals whose absolute values of second derivatives is $P$-convex and related fractional inequalities. J. Math. Inequal. 12(3), 655-664 (2018)

16. Hussain, S., Qaisar, S.: More results on Simpsons type inequality through convexity for twice differentiable continuous mappings. SpringerPlus 5, 77 (2016)

17. Iqbal, M., Qaisar, S., Hussain, S.: On Simpson's type inequalities utilizing fractional integral. J. Comput. Anal. Appl. 23(6), $1137-1145$ (2017)

18. Qaisar, S., Iqbal, M., Hussain, S., Butt, S.-I., Meraj, M.-A.: New inequalities on Hermite-Hadamard utilizing fractional integrals. Kragujev. J. Math. 42(1), 15-27 (2018)

19. Set, E., Ozdemir, M.-E., Uygun, N.: On new Simpson's type inequalities for quasi-convex functions via Riemann-Liouville integrals. AIP Conf. Proc. 1726, 020068 (2016)

20. Agarwal, P., Jleli, M., Tomar, M.: Certain Hermite-Hadamard type inequalities via generalized $k$-fractional integrals. J. Inequal. Appl. 2017, Article ID 55 (2017)

21. Kirane, M., Samet, B.: Discussion of some inequalities via fractional integrals. J. Inequal. Appl. 2018, Article ID 19 (2018)

22. Chu, Y.-M., Khan, M.-A., Ali, T., Dragomir, S.-S.: Inequalities for $\alpha$-fractional differentiable functions. J. Inequal. Appl. 2017, Article ID 93 (2017)

23. Wang, $H_{\text {., }} \mathrm{Du}, \mathrm{T}$., Zhang, Y.: $k$-fractional integral trapezium-like inequalities through $(h, m)$-convex and $(\alpha, m)$-convex mapping. J. Inequal. Appl. 2017, Article ID 311 (2017)

24. Ssooppy, N.-S.-K., Huang, C.-J., Rahman, G., Ghaffar, A., Qi, F.: Some inequalities of the Hermite-Hadamard type for $k$-fractional conformable integrals A. Aust. J. Math. Anal. Appl. 16(1), 1-9 (2019)

25. Qi, F., Habib, S., Mubeen, S., Naeem, N.-M.: Generalized k-fractional conformable integrals and related inequalities. AIMS Math. 4(3), 343-358 (2019)

\section{Submit your manuscript to a SpringerOpen ${ }^{\circ}$ journal and benefit from:}

- Convenient online submission

- Rigorous peer review

- Open access: articles freely available online

- High visibility within the field

- Retaining the copyright to your article

Submit your next manuscript at $\gg$ springeropen.com 